PROCEEDINGS OF THE

AMERICAN MATHEMATICAL SOCIETY

Volume 135, Number 9, September 2007, Pages 2669-2676

S 0002-9939(07)08892-2

Article electronically published on February 9, 2007

\title{
ON QUASIFREE PROFINITE GROUPS
}

\author{
LUIS RIBES, KATHERINE STEVENSON, AND PAVEL ZALESSKII
}

(Communicated by Jonathan I. Hall)

\begin{abstract}
Recently, it has been shown by Harbater and Stevenson that a profinite group $G$ is free profinite of infinite rank $m$ if and only if $G$ is projective and $m$-quasifree. The latter condition requires the existence of $m$ distinct solutions to certain embedding problems for $G$. In this paper we provide several new non-trivial examples of $m$-quasifree groups, projective and nonprojective. Our main result is that open subgroups of $m$-quasifree groups are $m$-quasifree.
\end{abstract}

A recent characterization of free profinite groups due to Harbater and Stevenson [1. Theorem 2.1] establishes that a profinite group $G$ is free profinite of infinite rank $m$ if and only if

(i) $G$ is projective, and

(ii) whenever one has a diagram

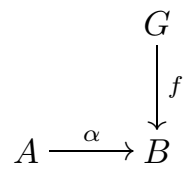

where $A$ and $B$ are finite groups, $f$ is an epimorphism of profinite groups, and $\alpha$ is an epimorphism of finite groups that splits and is not an isomorphism, there exist exactly $m$ different epimorphisms $\lambda: G \rightarrow A$ such that $\alpha \lambda=f$.

This builds on other well-known characterizations due to Iwasawa [3], Mel'nikov 4] and Chatzidakis [2] (see [5], Theorems 3.5.9 and 3.5.11 for a unified treatment in a slightly more general context).

In this paper we are interested in profinite groups that satisfy condition (ii) above. For an infinite cardinal $m$, we define a profinite group $G$ to be $m$-quasifree if it satisfies condition (ii) above. The following result of Harbater and Stevenson [1] provides naturally arising examples of $m$-quasifree groups which are not projective, and hence not free profinite.

Theorem 1 ([1, Theorem 1.1]). Let $k$ be a field and let $k((x, t))$ be the fraction field of the power series ring $k \llbracket x, t \rrbracket$, where $x$ and $t$ are indeterminates. Let $G=G_{k((x, t))}$ be the absolute Galois group of $k((x, t))$. Denote by $m$ the cardinality of $k((x, t))$. Then $G$ is an m-quasifree profinite group which is not projective.

Received by the editors May 3, 2006.

2000 Mathematics Subject Classification. Primary 20E18; Secondary 14G32.

The first author was partially supported by an NSERC grant.

The second author was partially supported by an NSF grant.

The third author was partially supported by CAPES and CNPq. 
In our main result (Theorem 2.1) we show that open subgroups of $m$-quasifree groups are $m$-quasifree. We also provide non-obvious examples of $m$-quasifree profinite groups.

\section{Preliminaries and examples}

Throughout this paper $\mathcal{C}$ denotes a variety of finite groups, i.e., a nonempty class of finite groups closed under the operations of taking subgroups, homomorphic images and finite direct products. For example $\mathcal{C}$ can be taken to be the class of all finite groups or the class of all finite solvable groups. A pro- $\mathcal{C}$ group is an inverse limit of groups in $\mathcal{C}$. We follow the notation and terminology of [5], where basic properties of these groups can be found.

Recall that an epimorphism $\alpha: A \longrightarrow B$ is said to split if there exists a homomorphism $\tau: B \longrightarrow A$ such that $\alpha \tau=\operatorname{id}_{B}$.

Definition 1.1. Let $\mathcal{C}$ be a variety of finite groups and let $m$ be an infinite cardinal. A pro- $\mathcal{C}$ group $Q$ is called an $m$-quasifree pro- $\mathcal{C}$ group if for every diagram of the form

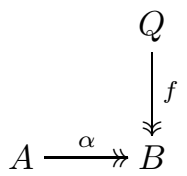

where $A$ and $B$ are finite groups, $f$ is an epimorphism of profinite groups, and $\alpha$ is an epimorphism of finite groups that splits and is not an isomorphism, there exist exactly $m$ different epimorphisms $\lambda: G \rightarrow A$ such that $\alpha \lambda=f$.

We refer to such a diagram as a split embedding problem of pro-C $\mathcal{C}$ groups for $Q$, and we say that an epimorphism $\lambda: Q \longrightarrow A$ such that $\alpha \lambda=f$ is a solution of the embedding problem. Hence $Q$ is $m$-quasifree if every finite split embedding problem has exactly $m$ different solutions.

Lemma 1.2. The minimal number of generators $d(Q)$ of an $m$-quasifree pro-C group $Q$ is $d(Q)=m$.

Proof. Recall that the local weight $w_{0}(Q)$ of an infinite profinite group $Q$ is the number of open normal subgroups of $Q$. Note that the minimal number of generators $d(Q)$ of $Q$ equals its local weight, $d(Q)=w_{0}(Q)$, since $Q$ is infinitely generated (see Proposition 2.6.2 in [5]). So it suffices to prove that $w_{0}(Q)=m$. For any open normal subgroup $N$ of $Q$, the number of continuous epimorphisms $\varphi_{N}: Q \longrightarrow Q / N$ with $N=\operatorname{Ker}\left(\varphi_{N}\right)$ is finite. Therefore for any finite group $A$, the number $n_{A}$ of open normal subgroups $N$ of $Q$ with $Q / N \cong A$ equals the number of continuous epimorphisms $Q \longrightarrow A$, which in turn equals $m$, because $Q$ is an $m$-quasifree group (just put $B=1$ in the embedding problem). Now

$$
w_{0}(Q)=\sum_{A} n_{A}=m \aleph_{0}=m,
$$

since the number of isomorphism classes of finite groups in $\mathcal{C}$ is $\aleph_{0}$.

Let $\mathcal{C}$ be a variety of finite groups. If $G$ is a profinite group, define $R_{\mathcal{C}}(G)$ to be the intersection of all closed normal subgroups $N$ of $G$ such that $G / N \in \mathcal{C}$. Then $G / R_{\mathcal{C}}(G)$ is the maximal pro- $\mathcal{C}$ quotient of $G$ (see [5], Section 3.4). The following result is clear. 
Proposition 1.3. Let $\mathcal{C}^{\prime} \subseteq \mathcal{C}$ be varieties of finite groups, and let $m$ be an infinite cardinal. If $Q$ is an $m$-quasifree pro-C group, then its maximal pro- $\mathcal{C}^{\prime}$ quotient $Q / R_{\mathcal{C}^{\prime}}(Q)$ is an m-quasifree pro- $\mathcal{C}^{\prime}$ group.

Proposition 1.4. Let $G$ be an $m$-quasifree pro-C group. Then $G$ contains a free pro-C group of countable rank.

Proof. We observe (see [5], Corollary 2.6.6) that if $H$ is a pro- $\mathcal{C}$ group that admits a countable set of generators converging to 1 , then $H$ contains a countable collection of open normal subgroups

$$
H=U_{0}>U_{1}>\cdots
$$

that form a fundamental system of neighborhoods of 1 , and so

$$
H=\lim _{i \in I} H / U_{i} \leq \prod_{i} H / U_{i}
$$

It follows that $H$ appears as a closed subgroup of the cartesian product of the set of all finite groups in $\mathcal{C}$. In particular the free pro- $\mathcal{C}$ group $F$ of countable rank appears as a closed subgroup of such a cartesian product.

Therefore to prove the proposition it is enough to construct an epimorphism $\lambda: G \longrightarrow \prod_{i=0}^{\infty} K_{i}$, where $K_{i}$ runs over all finite groups in $\mathcal{C}$, where we assume $K_{0}=1$. To do this we construct inductively compatible epimorphisms

$$
\lambda_{n}: G \longrightarrow \prod_{i=0}^{n} K_{i} .
$$

If $\lambda_{n-1}$ has been constructed, consider the split embedding problem

$$
\begin{aligned}
& \left.\right|^{G} \lambda_{n-1} \\
& \prod_{i=0}^{n} K_{i} \stackrel{\alpha_{n}}{\longrightarrow} \prod_{i=1}^{n-1} K_{i}
\end{aligned}
$$

where $\alpha_{n}$ is the natural projection. Since $G$ is quasifree, there exists an epimorphism $\lambda_{n}: G \longrightarrow \prod_{i=0}^{n} K_{i}$ such that $\alpha_{n} \lambda_{n}=\lambda_{n-1}(n=1,2, \ldots)$. The inverse limit of these maps

$$
\lambda=\lim _{n} \lambda_{n}: G \longrightarrow \prod_{i=0}^{\infty} K_{i}
$$

provides the required epimorphism.

If $A$ and $B$ are pro- $\mathcal{C}$ groups, we denote by $A \amalg B$ their free pro- $\mathcal{C}$ product, i.e., their coproduct in the category of pro- $\mathcal{C}$ groups (see [5], Section 9.1). For simplicity and to avoid the concept of a free pro- $\mathcal{C}$ product of groups indexed by a profinite space, we state part (b) of the following lemma only for finite groups $A$ and $B$, but the result is valid in general (in fact it follows by making more detailed the argument given for part (c)). One says that a variety of finite groups $\mathcal{C}$ is extension closed if whenever $1 \rightarrow K \rightarrow G \rightarrow H \rightarrow 1$ is an exact sequence of finite groups such that $K, H \in \mathcal{C}$, then $G \in \mathcal{C}$.

Lemma 1.5. Assume that the variety of finite groups $\mathcal{C}$ is extension closed. Let $G=A \amalg B$ be a free pro-C product of two pro-C groups $A$ and $B$. Let $A^{G}$ denote the smallest closed normal subgroup of $G$ generated by $A$. 
(a) Let $\varphi: G=A \amalg B \longrightarrow B$ be the continuous epimorphism that sends $A$ to 1 and is the identity on $B$. Let $K=\operatorname{ker}(\varphi)$. Then $K=A^{G}$ and $G / A^{G} \cong B$.

(b) If $A$ and $B$ are finite, then $A^{G}$ is the free pro-C product of the subgroups $\left\{A^{b}=b^{-1} A b \mid b \in B\right\}$ of $G$.

(c) $A^{G}$ is topologically generated by $\left\{A^{b} \mid b \in B\right\}$.

Proof. (a) Since $K \cap B=1$ and $K B=G$, it follows that $G=K \rtimes B$. Obviously $A^{G} \leq K$. So $G=A^{G} \rtimes B$. Thus $A^{G}=K$ and clearly $G / A^{G} \cong B$.

(b) This follows from the analog of the Kurosh subgroup theorem for free products of pro- $\mathcal{C}$ groups. Indeed, observe that $K=A^{G}$ is a normal open subgroup of $G=A \amalg B$ with $G / K \cong B$. Then (see [5], Theorem 9.1.9)

$$
K=\left[\coprod_{\tau \in K \backslash G / A} K \cap g_{\tau} A g_{\tau}^{-1}\right] \amalg\left[\coprod_{\nu \in K \backslash G / B} K \cap g_{\nu}^{\prime} B g_{\nu}^{\prime-1}\right] \amalg F,
$$

where $g_{\tau}$ ranges through a set of representatives of the double cosets $K \backslash G / A$ and $g_{\nu}^{\prime}$ ranges through a set of representatives of the double cosets $K \backslash G / B$, and where $F$ is a free pro-C group of rank $1+[G: K]-|K \backslash G / A|-|K \backslash G / B|$. In our case, since $K \triangleleft G, K \geq A$ and $G / K \cong B$, it follows that $\operatorname{rank}(F)=0$ and $K=\coprod_{b \in B} A^{b}$, the free pro- $\mathcal{C}$ product of the conjugates $A^{b}$ of $A$ by the elements of $B$.

(c) Let $\mathcal{U}$ be the collection of all open normal subgroups of $G$. For $U \in \mathcal{U}$, put $A_{U}=A / A \cap U, B_{U}=B / B \cap U$ and $G_{U}=A_{U} \amalg B_{U}$. Then

$$
G={\underset{\lim }{U \in \mathcal{U}}}_{\lim _{U}} G_{\text {. }}
$$

Note that

$$
A^{G}={\underset{U \in U}{\lim }}_{A_{U}}^{G_{U}}
$$

By part (b), $A_{U}^{G_{U}}$ is topologically generated by $\left\{A_{U}^{b_{U}} \mid b_{U} \in B_{U}\right\}$. Hence the result follows, since

$$
A=\lim _{U \in \mathcal{U}} A_{U} \quad \text { and } \quad B=\lim _{U \in \mathcal{U}} B_{U}
$$

Examples 1.6. 1. Let $m$ be an infinite cardinal. A free profinite group $F=F(m)$ of rank $m$ is $m$-quasifree. In fact a profinite group is free profinite of rank $m$ if and only if it is $m$-quasifree and projective.

2. (D. Haran) If $Q$ is an $m$-quasifree group and $H$ is a profinite group with $d(H) \leq m$, then their free profinite product $Q \amalg H$ is $m$-quasifree.

3 . Let $F$ be a free profinite group on a countable set of generators $x_{1}, y_{1}, x_{2}, y_{2}, \ldots$ convergent to 1 . Observe that the infinite product $\left[x_{1}, y_{1}\right]\left[x_{2}, y_{2}\right] \cdots$ converges in $F$ and so it defines a unique element $r$. Define a profinite group $G$ imposing on $F$ the relation $\left[x_{1}, y_{1}\right]\left[x_{2}, y_{2}\right] \cdots$, i.e., $G=F /(r)$, where $(r)$ denotes the smallest closed normal subgroup of $F$ containing $r$.

We shall show that $G$ is $\aleph_{0}$-quasifree. Consider a split embedding problem

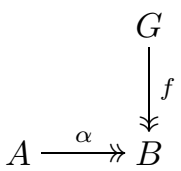


Put $K=\operatorname{Ker}(\alpha)$. Let $\theta: B \longrightarrow A$ be a homomorphism such that $\alpha \theta=\mathrm{id}_{B}$. Then $A=K \rtimes \theta(B)$. Since $B$ is finite, there exists a natural number $t$ such that $f\left(x_{j}\right)=f\left(y_{j}\right)=1$, for all $j>t$. Let $k_{1}, \ldots, k_{n}$ be the elements of $K$.

Next we define an infinite countable set of continuous epimorphism $\left\{\eta_{s}: F \longrightarrow\right.$ $A \mid s=0,1,2, \ldots\}$. The epimorphism $\eta_{s}$ is determined by

$$
\eta_{s}\left(x_{j}\right)= \begin{cases}(\theta f)\left(x_{j}\right), & \text { if } 1 \leq j \leq t+s, \\ k_{i}, & \text { if } j=t+s+i, i=1, \ldots, n, \\ 1 & \text { if } j>t+s+n .\end{cases}
$$

and

$$
\eta_{s}\left(y_{j}\right)= \begin{cases}(\theta f)\left(y_{j}\right), & \text { if } 1 \leq j \leq t+s, \\ k_{i}, & \text { if } j=t+s+i, i=1, \ldots, n, \\ 1 & \text { if } j>t+s+n .\end{cases}
$$

Observe that $\eta_{s}(r)=1$. Therefore, $\eta_{s}$ induces a continuous epimorphism $\lambda_{s}$ : $G \longrightarrow A$. Moreover $\lambda_{s} \neq \lambda_{s^{\prime}}$, if $s \neq s^{\prime}$, and $\alpha \lambda_{s}=f$, for all $s=0,1,2, \ldots$ Since $d(G)=\aleph_{0}$, this shows that the above embedding problem has exactly $\aleph_{0}$ solutions.

4. It is easy to generalize example 3 to an infinite family of examples of the same type, for example choose $r=\prod_{i=1}^{\infty}\left[x_{i}^{2}, y_{i}^{2}\right]$.

\section{Open SUBGROUPS OF QUASIFREE GROUPS}

Theorem 2.1. Assume that $\mathcal{C}$ is an extension closed variety of finite groups. Let $H$ be an open subgroup of an $m$-quasifree pro-C group $G$. Then $H$ is $m$-quasifree.

Proof. Consider the following split embedding problem of pro- $\mathcal{C}$ groups for $H$

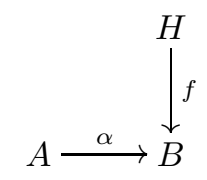

Note that the groups $A$ and $B$ are finite groups as $\mathcal{C}$ denotes a variety of finite groups. We shall prove first that this embedding problem has at least one solution.

Put $K=\operatorname{Ker}(\alpha)$, and let $T=\operatorname{Ker}(f)_{G}$ denote core of the subgroup $\operatorname{Ker}(f)$ in $G$, that is, the intersection of all conjugates of $\operatorname{Ker}(f)$ in $G$. Then $T$ is open and normal in $G$. Let $\beta: G \longrightarrow B^{\prime}=G / T$ be the canonical epimorphism and define $B_{H}^{\prime}=\beta(H)=H / T$. Denote by

$$
\bar{f}: B_{H}^{\prime}=H / T \longrightarrow B
$$

the natural map induced by $f$.

Construct the free pro- $\mathcal{C}$ product $A^{\prime}=K \amalg B^{\prime}$ of $K$ and $B^{\prime}$. By Lemma 1.5, the closed normal subgroup $\tilde{K}$ of $A^{\prime}$ generated by $K$ is the free pro- $\mathcal{C}$ product $\tilde{K}=\coprod_{b \in B^{\prime}} K^{b}$. Note that

$$
A^{\prime}=K \amalg B^{\prime}=\tilde{K} \rtimes B^{\prime} .
$$

Consider the open subgroup

$$
L=\tilde{K} \rtimes B_{H}^{\prime}
$$

of $A^{\prime}$.

Observe that the subgroup $B_{H}^{\prime}$ of $L$ normalizes the free factors $\coprod_{b \in B_{H}^{\prime}} K^{b}$ and $\coprod_{b \in\left(B^{\prime}-B_{H}^{\prime}\right)} K^{b}$ of $\tilde{K}=\coprod_{b \in B^{\prime}} K^{b}=\left(\coprod_{b \in B_{H}^{\prime}} K^{b}\right) \amalg\left(\coprod_{b \in\left(B^{\prime}-B_{H}^{\prime}\right)} K^{b}\right)$. It follows 
from Lemma 1.5 that the closed normal subgroup of $\tilde{K}$ generated by $\coprod_{b \in\left(B^{\prime}-B_{H}^{\prime}\right)} K^{b}$ is normalized by $B_{H}^{\prime}$, and therefore it is normal in $L$. Thus there is a natural epimorphism

$$
\gamma: L \longrightarrow\left(\coprod_{b \in B_{H}^{\prime}} K^{b}\right) \rtimes B_{H}^{\prime}=K \amalg B_{H}^{\prime}
$$

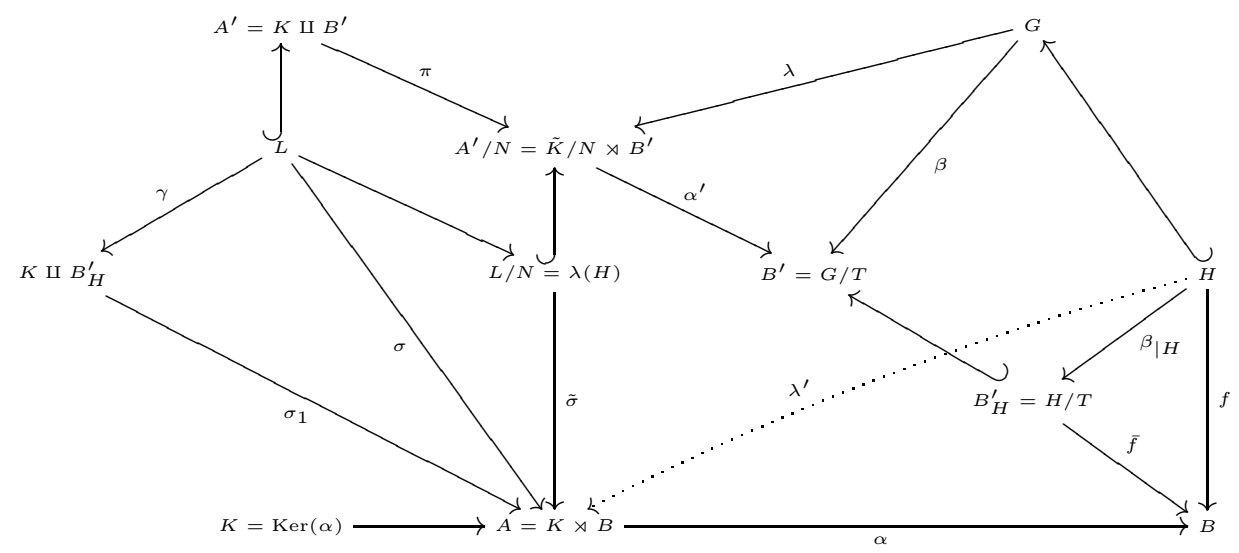

Let

$$
\sigma_{1}: K \amalg B_{H}^{\prime} \longrightarrow A=K \rtimes B
$$

be the continuous epimorphism induced by the identity map $K \longrightarrow K$ and the map $\bar{f}: B_{H}^{\prime} \longrightarrow B$. Put $\sigma=\sigma_{1} \gamma$.

Define

$$
N=(\operatorname{Ker}(\sigma) \cap \tilde{K})_{A^{\prime}}
$$

the core of $\operatorname{Ker}(\sigma) \cap \tilde{K}$ in $A^{\prime}$, so that $N$ is open normal in $A^{\prime}$ and contained in $\operatorname{Ker}(\sigma) \cap \tilde{K}$. Observe that $N \cap B^{\prime}=N \cap K=1$. Consider the finite group

$$
A^{\prime} / N=(\tilde{K} / N) \rtimes B^{\prime}
$$

Let

$$
\pi: A^{\prime} \longrightarrow A^{\prime} / N \quad \text { and } \quad \alpha^{\prime}: A^{\prime} / N \longrightarrow B^{\prime}
$$

be the canonical epimorphisms. Since $G$ is $m$-quasifree and $\alpha^{\prime}$ is an epimorphism of finite groups which splits, there exists an epimorphism

$$
\lambda: G \longrightarrow A^{\prime} / N
$$

such that $\alpha^{\prime} \lambda=\beta$. Since $N \leq \operatorname{Ker}(\sigma)$, we deduce that $\sigma$ factors through $L / N=$ $\pi(L)$. Let $\tilde{\sigma}: L / N \longrightarrow A$ be the map induced by $\sigma$.

We claim that $L / N=\lambda(H)$. To see this it suffices to show that $\pi^{-1}(\lambda(H))=L$. We show first that

$$
\lambda(H)=\alpha^{\prime-1}(\beta(H)) .
$$

Since $\beta(H)=\alpha^{\prime}(\lambda(H))$, we clearly have that $\lambda(H) \leq \alpha^{\prime-1}(\beta(H))$. For the reverse inclusion, note that

$$
\begin{aligned}
{[G: H] } & \geq[\lambda(G): \lambda(H)] \geq\left[\alpha^{\prime-1}\left(B^{\prime}\right): \alpha^{\prime-1}(\beta(H))\right] \\
& =\left[B^{\prime}: \beta(H)\right]=\left[B^{\prime}: B_{H}^{\prime}\right]=[G: H]
\end{aligned}
$$

Hence

$$
\lambda(H)=\alpha^{\prime-1}(\beta(H))=(\tilde{K} / N) \rtimes B_{H}^{\prime},
$$


as desired. Therefore,

$$
\lambda(H) \geq \operatorname{Ker}\left(\alpha^{\prime}\right)=\tilde{K} / N
$$

and so,

$$
\pi^{-1}(\lambda(H)) \geq \tilde{K}
$$

Since obviously $\pi^{-1}(\lambda(H)) \geq B_{H}^{\prime}$, we deduce that $\pi^{-1}(\lambda(H)) \geq L=\tilde{K} \rtimes B_{H}^{\prime}$. If $\pi^{-1}(\lambda(H)) \neq L$, then $\pi^{-1}(\lambda(H))$ would contain elements of $B^{\prime}-B_{H}^{\prime}$, and so

$$
B_{H}^{\prime}=\beta(H)=\left(\alpha^{\prime} \lambda\right)(H)=\left(\alpha^{\prime} \pi \pi^{-1} \lambda\right)(H) \neq B_{H}^{\prime},
$$

a contradiction. Thus $\pi^{-1}(\lambda(H))=L$, proving the claim.

Next define $\lambda^{\prime}=\tilde{\sigma} \lambda_{\mid H}$. We now check that $\alpha \lambda^{\prime}=f$. Indeed, $\alpha^{\prime}(L / N) \leq B_{H}^{\prime}$. On the other hand, since $L=\tilde{K} \rtimes B_{H}^{\prime}$, we have $L / N=(\tilde{K} / N) \rtimes B_{H}^{\prime}$. Note that $\alpha \tilde{\sigma}_{\mid B_{H}^{\prime}}=\bar{f}$. So $\alpha \tilde{\sigma}=\bar{f} \alpha_{\mid L / N}^{\prime}$. Hence $\alpha \lambda^{\prime}=\bar{f} \alpha^{\prime} \lambda_{\mid H}=\bar{f} \beta_{\mid H}=f$, as needed.

To finish the proof that $H$ is $m$-quasifree, we must verify that the above split embedding problem has exactly $m$ solutions. The number of maps $\lambda$ in the diagram above is $m$, since $G$ is $m$-quasifree. Since $m$ is infinite and the index of $H$ in $G$ is finite, the number of $\lambda^{\prime}$ that can be obtained by the construction above is $m$. So the total number of solutions of the diagram

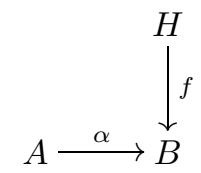

is at least $m$. But obviously the total number of solutions is at most $d(H)=d(G)$. By Lemma 1.2, $d(G)=m$. Thus the total number of solutions is $m$.

\section{ACKNOWLEDGMENTS}

This research was initiated while we were participating in the meeting 'Field Arithmetic' at Oberwolfach. We thank the organizers of the meeting and the Mathematisches Forschunginstitut for providing us the opportunity to work together. Finally, the authors would like to thank David Harbater for pointing out an imprecision in the proof of Theorem 2.1 and for a useful suggestion to clarify it.

\section{REFERENCES}

[1] D. Harbater and K. Stevenson, Local Galois theory in dimension two, Advances in Mathematics, 198 (2) (2005) 623-653. MR2183390

[2] Z. A. Chatzidakis, Model theory of profinite groups having the Iwasawa property, Illinois J. Math., 42 (1998) 70-96. MR.1492040 (99j:03027)

[3] I. Iwasawa, On solvable extensions of algebraic number fields, Ann. Math., 58 (1953) 548572. MR0059314 (15,509d)

[4] O. V. Mel'nikov, Normal subgroups of free profinite groups, Izv. Akad. Nauk, 42, 3-25. English transl.: Math. USSR Izvestija, 12 (1978) 1-20. MR0495682 (80d:20028)

[5] L. Ribes and P. Zalesskii, Profinite Groups, Ergebn. der Mathematik, Vol. 40, SpringerVerlag, Berlin 2000. MR,1775104 (2001k:20060) 
School of Mathematics and Statistics, Carleton University, Ottawa, Ontario, CANADA K1S 5B6

E-mail address: lribes@math.carleton.ca

Department of Mathematics, California State University Northridge, Northridge, CAlifornia 91330

E-mail address: katherine.stevenson@csun.edu

Department of Mathematics, University of Brasília, Brasília, Brazil

E-mail address: pz@mat.unb.br 